\title{
Computationally Efficient 2D DOA Estimation for L-Shaped Array with Unknown Mutual Coupling
}

\author{
Yang-Yang Dong $(\mathbb{D}$ and Xin Chang \\ School of Electronic Engineering, Xidian University, Xian, Shaanxi 710071, China \\ Correspondence should be addressed to Yang-Yang Dong; dongyangyang2104@126.com
}

Received 9 November 2017; Revised 26 December 2017; Accepted 9 January 2018; Published 31 January 2018

Academic Editor: Raffaele Solimene

Copyright (c) 2018 Yang-Yang Dong and Xin Chang. This is an open access article distributed under the Creative Commons Attribution License, which permits unrestricted use, distribution, and reproduction in any medium, provided the original work is properly cited.

\begin{abstract}
Although L-shaped array can provide good angle estimation performance and is easy to implement, its two-dimensional (2D) direction-of-arrival (DOA) performance degrades greatly in the presence of mutual coupling. To deal with the mutual coupling effect, a novel 2D DOA estimation method for L-shaped array with low computational complexity is developed in this paper. First, we generalize the conventional mutual coupling model for L-shaped array and compensate the mutual coupling blindly via sacrificing a few sensors as auxiliary elements. Then we apply the propagator method twice to mitigate the effect of strong source signal correlation effect. Finally, the estimations of azimuth and elevation angles are achieved simultaneously without pair matching via the complex eigenvalue technique. Compared with the existing methods, the proposed method is computationally efficient without spectrum search or polynomial rooting and also has fine angle estimation performance for highly correlated source signals. Theoretical analysis and simulation results have demonstrated the effectiveness of the proposed method.
\end{abstract}

\section{Introduction}

Two-dimensional (2D) direction-of-arrival (DOA) estimation is an important area of array signal processing and has wide applications in wireless communication, radar, sonar, electronic warfare, and so far $[1,2]$. In order to obtain unambiguous angle estimation, many researchers often restrict the minimum sensor spacing to not more than half-wavelength, which results in the mutual coupling effect $[3,4]$. Various researches have indicated that mutual coupling changes the array manifold matrix and causes severe angle estimation performance degradation $[5,6]$. To mitigate the mutual coupling effect, researchers have developed many 2D DOA estimation methods with unknown mutual coupling [3-14]. These methods can mainly be classified into three types: electromagnetic simulation $[3,4,6]$, active calibration $[7,8]$, and blind calibration [5, 9-14]. The electromagnetic simulation methods in $[3,4]$ use the electromagnetic theory to calculate the mutual coupling and are high in accuracy. What is more, the mutual coupling coefficients are often easy to be affected by the environment and cannot be as stable as a constant, which causes the fact that this kind of methods cannot be used for the application fields with drastic change on environment, such as antiradiation missile (ARM) [2]. Meanwhile, for active calibration methods [7, 8], they require at least one calibration source to estimate the mutual coupling, where the additional active calibration sources are often not available in practice [9].

While for blind calibration algorithms, they are very promising since there is no requirement for stable environment or calibration sources and 2D DOAs in the presence of unknown mutual coupling can be estimated only via signal processing. There are three primary techniques to deal with the unknown mutual coupling. The first one is realized by using iterative procedure to compensate the mutual coupling and estimate 2D DOAs [5], which can be applied for many array geometries. However, it sometimes converges slowly and may cause the increase in runtime and even wrong DOA estimations [9]. The second one is called RAnk REduction (RARE) technique $[9,12,14]$, which exploits the special structure of coupled array manifold matrix to construct a cost function similar to that of MUSIC method and estimate the angles via minimizing the cost function. However, the angle estimation via RARE technique often needs 
multidimensional search, which is still high in computational complexity [14]. The third technique is named auxiliary sensor technique, which blindly compensates the mutual coupling at the cost of a few auxiliary sensors and generates new received array data $[10,13]$. Since the data often possesses special structure and is much simpler compared to the RARE technique, many computationally efficient 2D DOA estimation methods available can be applied via a few simple modifications. Therefore, compared to the other two techniques, the auxiliary sensor technique is more computationally efficient.

The blind calibration methods above are developed for various 2D arrays. Although Rübsamen and Gershman have developed novel sparse $2 \mathrm{D}$ arrays which can provide nonambiguous DOA estimates for the full $360^{\circ}$ azimuth field-of-view [15], the structures of the sparse 2D arrays are too complicated for hardware manufacturers to implement. Among the common arrays for 2D DOA estimation available and in consideration of the angle estimation performance and implementability, L-shaped array is of the optimal implementability [16]. Hence, 2D DOA estimation for L-shaped array with unknown mutual coupling is essential. Wu et al. [12] have proposed a RARE technique based method, which requires two-dimensional search and is still too high in computational complexity for real-time applications [2]. Luckily, Liang et al. [13] have developed an auxiliary sensor based method, which can estimate $2 \mathrm{D}$ angles in the presence of mutual coupling without spectrum search or pair matching, which is much lower than Wu et al.s method [12] in terms of computational complexity. However, Liang et al's method has three main drawbacks: (1) it can only hold for the case that the maximum mutual coupling degree is equal to 2 , which restricts its application in more general cases; (2) it cannot deal with highly correlated source signals, which is an unavoidable problem for many real applications, such as the electronic reconnaissance with deception jamming [2]; (3) it divides the 2D DOA estimation into two sequential steps, which causes the error accumulation effect and has a bad effect on angle estimations.

To handle the above problems, in this paper, we first generalize the data model for any mutual coupling degree. Inspired by Liang et al.'s method [13], the proposed method obtains the new received array data via auxiliary sensor technique mentioned above. Then, we utilize the propagator method twice to decrease the source signal correlation and form two matrices associated with 2D DOAs which have the same eigenvectors and the different eigenvalues. Finally, the 2D DOAs are estimated simultaneously via the complex eigenvalue technique [17]. Simulation experiments and theoretical analysis have demonstrated the validity of the proposed method and proved that it can handle the three problems encountered in Liang et al's method [13]. What is more, compared with Wu et al's method [12] and Liang et al's method [13], the proposed method can handle the angle estimation for highly correlated source signals well with lower computational complexity.

Notations. Matrices and vectors are denoted by boldfaced capital letters and lowercase letters, respectively. The superscripts $(\cdot)^{*},(\cdot)^{T},(\cdot)^{H},(\cdot)^{-1}$, and $(\cdot)^{\dagger}$ stand for conjugate,

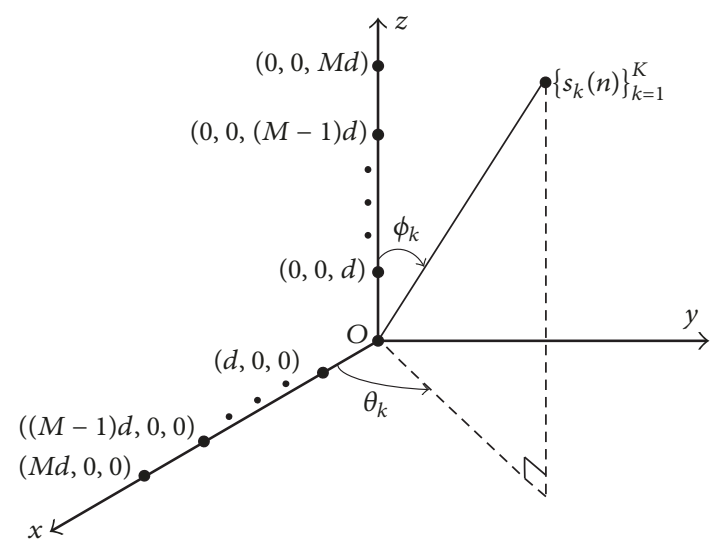

FIGURE 1: L-shaped array configuration for 2D DOA estimation.

transpose, conjugate transpose, inverse, and pseudoinverse. The notations $E\{\cdot\}, \otimes, \operatorname{var}(\cdot), \mathbf{I}_{D}, \mathbf{J}_{D}, \mathbf{0}_{m \times n}, \operatorname{diag}\{\cdot\}, \operatorname{Re}\{\cdot\}$, and $\operatorname{Im}\{\cdot\}$ denote the statistical expectation, Kronecker product, the variance of a random variable, a $D \times D$ identity matrix, a $D \times D$ exchange matrix with the ones on its antidiagonal and zeros elsewhere, an $m \times n$ zero matrix, diagonalization, and the real and imaginary part of a complex number, respectively.

\section{Signal Model}

Two uniform linear orthogonal arrays with intersensor spacing $d$ form an $x-z$ plane L-shaped array configuration as shown in Figure 1 and the total number of sensors is $2 M+$ 1 . We assume $K$ noncoherent narrowband far-field signals $\left\{s_{k}(n)\right\}_{k=1}^{K}(n=1,2, \ldots, N$, where $N$ denotes the number of snapshots) of wavelength $\lambda$ from distinct directions with azimuth and elevation angles $\left\{\left(\theta_{k}, \phi_{k}\right)\right\}_{k=1}^{K}\left(0^{\circ} \leq \theta_{k} \leq 180^{\circ}\right.$, $\left.0^{\circ} \leq \phi_{k} \leq 180^{\circ}\right)$ impinging on the L-shaped array.

Let $\alpha_{k}=-2 \pi d \cos \theta_{k} \sin \phi_{k} / \lambda$ and $\beta_{k}=-2 \pi d \cos \phi_{k} / \lambda$; the received array data vector at the $n$th snapshot can be expressed as [13]

$$
\begin{aligned}
\mathbf{y}(n) & =\mathbf{C A s}(n)+\mathbf{w}(n) \\
& =\sum_{k=1}^{K} \mathbf{C a}\left(\alpha_{k}, \beta_{k}\right) s_{k}(n)+\mathbf{w}(n),
\end{aligned}
$$

where $\mathbf{C}, \mathbf{A}, \mathbf{a}\left(\alpha_{k}, \beta_{k}\right), \mathbf{s}(n)$, and $\mathbf{w}(n)$ represent the unknown mutual coupling matrix, ideal manifold matrix, ideal steering vector, source signal vector, and noise vector, respectively. In detail, $\mathbf{y}(n)=$ $\left[y_{1,0}(n) \cdots y_{M, 0}(n) y_{0,0}(n) y_{0,1}(n) \cdots y_{0, M}(n)\right]^{T}, \mathbf{A}=\left[\mathbf{a}\left(\alpha_{1}, \beta_{1}\right)\right.$, $\left.\mathbf{a}\left(\alpha_{2}, \beta_{2}\right), \ldots, \mathbf{a}\left(\alpha_{K}, \beta_{K}\right)\right], \mathbf{a}\left(\alpha_{k}, \beta_{k}\right)=\left[e^{j \alpha_{k}}, \ldots, e^{j M \alpha_{k}}, 1\right.$, $\left.e^{j \beta_{k}}, \ldots, e^{j M \beta_{k}}\right]^{T}, \mathbf{s}(n)=\left[s_{1}(n), s_{2}(n), \ldots, s_{K}(n)\right]^{T}, \mathbf{w}(n)=$ $\left[w_{1,0}(n) \cdots w_{M, 0}(n) w_{0,0}(n) w_{0,1}(n) \cdots w_{0, M}(n)\right]^{T}$.

In [13], $\mathbf{C}$ is constructed via assuming that each sensor is only affected by its adjacent sensors within the distance of $\sqrt{5} d$, which is not general. To construct a more general mutual coupling model for L-shaped array, we firstly define the maximum coupling range as $p d$ ( $p$ is a positive integer). Then, with the fact that the mutual coupling matrix of ULA 
can be modeled as a banded symmetric Toeplitz matrix, $\mathbf{C}$ can be expressed as a block symmetric matrix

$$
\mathbf{C}=\left[\begin{array}{lll}
\mathbf{F} & \mathbf{g} & \mathbf{H} \\
\mathbf{g}^{T} & 1 & \mathbf{g}^{T} \\
\mathbf{H} & \mathbf{g} & \mathbf{F}
\end{array}\right],
$$

where the mutual coupling matrix within each subarray is $\mathbf{F}=$ Toeplitz $\left\{\left[1, c_{1}, \ldots, c_{p}, \mathbf{0}_{1 \times(\mathrm{M}-p-1)}\right]\right\}$, the mutual coupling matrix corresponding to the element at the origin is $\mathbf{g}=$ $\left[c_{1}, \ldots, c_{p}, \mathbf{0}_{1 \times(M-p)}\right]^{T}$, the mutual coupling matrix between two subarrays is $\left\{\mathbf{H}_{l, m}=\mathbf{H}_{m, l}, \sqrt{m^{2}+l^{2}} \leq p ; \mathbf{H}_{l, m}=\right.$ $\left.0, \sqrt{m^{2}+l^{2}}>p\right\}$. (The subscript $(m, l)$ stands for the sensor with coordinates $(m d, 0, l d)$.)

\section{Proposed Method}

3.1. Blind Mutual Coupling Effect Compensation. Inspired by the analysis done in [13], let us define two selection matrices firstly

$$
\begin{aligned}
& \mathbf{T}_{1}=\left[\mathbf{0}_{(M+1-2 p) \times(p-1)} \mathbf{I}_{M+1-2 p} \mathbf{0}_{(M+1-2 p) \times(M+1+p)}\right], \\
& \mathbf{T}_{2}=\left[\mathbf{0}_{(M+1-2 p) \times(M+p)} \mathbf{I}_{M+1-2 p} \mathbf{0}_{(M+1-2 p) \times p}\right],
\end{aligned}
$$

where $\mathbf{T}_{1}$ and $\mathbf{T}_{2}$ satisfy $\mathbf{T}_{1} \mathbf{T}_{1}^{H}=\mathbf{I}_{M+1-2 p}$ and $\mathbf{T}_{1} \mathbf{T}_{2}^{H}=$ $\mathbf{0}_{(M+1-2 p) \times(M+1-2 p)}$.

Applying $\mathbf{T}_{1}$ to (1), we have

$$
\mathbf{x}(n)=\mathbf{T}_{1} \mathbf{y}(n)=\mathbf{A}_{x} \boldsymbol{\Lambda}_{x} \mathbf{s}(n)+\mathbf{w}_{x}(n)
$$

where $\mathbf{A}_{x}=\left[\mathbf{a}_{x}\left(\alpha_{1}\right), \mathbf{a}_{x}\left(\alpha_{2}\right), \ldots, \mathbf{a}_{x}\left(\alpha_{K}\right)\right]$ and $\mathbf{a}_{x}\left(\alpha_{k}\right)=$ $\left[1, e^{j \alpha_{k}}, \ldots, e^{j(M-2 p) \alpha_{k}}\right]^{T} . \Lambda_{x}=\operatorname{diag}\left\{\gamma_{1}^{x}, \gamma_{2}^{x}, \ldots, \gamma_{K}^{x}\right\}$ and $\gamma_{k}^{x}=$ $e^{j p \alpha_{k}}+\sum_{l=1}^{p}\left[c_{p-l+1} e^{j(l-1) \alpha_{k}}+c_{l} e^{j(p+l) \alpha_{k}}\right]$.

Similarly, applying $\mathbf{T}_{2}$ to (1), we can obtain

$$
\mathbf{z}(n)=\mathbf{T}_{2} \mathbf{y}(n)=\mathbf{A}_{z} \boldsymbol{\Lambda}_{z} \mathbf{s}(n)+\mathbf{w}_{z}(n),
$$

where $\mathbf{A}_{z}=\left[\mathbf{a}_{z}\left(\beta_{1}\right), \mathbf{a}_{z}\left(\beta_{2}\right), \ldots, \mathbf{a}_{z}\left(\beta_{K}\right)\right]$ and $\mathbf{a}_{z}\left(\beta_{k}\right)=\left[1, e^{j \beta_{k}}\right.$, $\left.\ldots, e^{j(M-2 p) \beta_{k}}\right]^{T} . \Lambda_{z}=\operatorname{diag}\left\{\gamma_{1}^{z}, \gamma_{2}^{z}, \ldots, \gamma_{K}^{z}\right\}$ and $\gamma_{k}^{z}=e^{j p \beta_{k}}+$ $\sum_{l=1}^{p}\left[c_{p-l+1} e^{j(l-1) \beta_{k}}+c_{l} e^{j(p+l) \beta_{k}}\right]$.

Hence, the mutual coupling effect is compensated blindly at the cost of a few auxiliary sensors.

3.2. Joint Azimuth and Elevation Angle Estimation. With (4) and (5), Liang et al's method in [13] can be applied with a few modifications. However, Liang et al.'s method cannot handle source signals with moderate or high correlation and has the error accumulation effect for 2D DOA estimations.

To solve the problems encountered in [13], we firstly apply the propagator method (PM) [18] to the new received array data $\widetilde{\mathbf{y}}(n)=\left[\mathbf{x}^{T}(n), \mathbf{z}^{T}(n)\right]^{T}$ and obtain its corresponding propagator matrix $\mathbf{P}$ as follows:

$$
\mathbf{P}=\left(\widetilde{\mathbf{R}}_{1}^{H} \widetilde{\mathbf{R}}_{1}\right)^{-1} \widetilde{\mathbf{R}}_{1}^{H} \widetilde{\mathbf{R}}_{2},
$$

where $\widetilde{\mathbf{R}}_{1}$ and $\widetilde{\mathbf{R}}_{2}$ are two parts of the covariance matrix $\widetilde{\mathbf{R}}=E\left\{\widetilde{\mathbf{y}}(n) \widetilde{\mathbf{y}}^{H}(n)\right\}=\widetilde{\mathbf{A}} \mathbf{R}_{s s} \widetilde{\mathbf{A}}^{H}+\sigma_{w}^{2} \mathbf{I}_{2 M-4 p+2}=\left[\widetilde{\mathbf{R}}_{1}, \widetilde{\mathbf{R}}_{2}\right]$ and $\widetilde{\mathbf{R}}_{1}$ consists of first $K$ columns of $\widetilde{\mathbf{R}}$. Additionally, $\mathbf{R}_{s s}=$ $E\left\{\mathbf{s}(n) \mathbf{s}^{H}(n)\right\}$ and $\widetilde{\mathbf{A}}=\left[\left(\mathbf{A}_{x} \boldsymbol{\Lambda}_{x}\right)^{T},\left(\mathbf{A}_{z} \boldsymbol{\Lambda}_{z}\right)^{T}\right]^{T}$.

Then, to exploit all array information available from $\widetilde{\mathbf{y}}(n)$, we define a modified propagator matrix $\mathbf{P}_{m}=\left[\mathbf{I}_{K}, \mathbf{P}\right]^{H}$ satisfying

$$
\mathbf{P}_{m} \widetilde{\mathbf{A}}_{1}=\widetilde{\mathbf{A}},
$$

where $\widetilde{\mathbf{A}}=\left[\widetilde{\mathbf{A}}_{1}^{T}, \widetilde{\mathbf{A}}_{2}^{T}\right]^{T} . \widetilde{\mathbf{A}}_{1}$ and $\widetilde{\mathbf{A}}_{2}$ are the first $K$ and the last $(2 M-4 p+2-K)$ rows of $\widetilde{\mathbf{A}}$.

To make full use of the potential rotational invariance property in $\mathbf{P}_{m}$, carry out the following calculations:

$$
\begin{aligned}
& \mathbf{Q}_{1}=\left(\mathbf{G}_{3} \mathbf{P}_{m}\right)\left(\mathbf{G}_{1} \mathbf{P}_{m}\right)^{\dagger}=\widetilde{\mathbf{A}}_{z 1} \boldsymbol{\Lambda}_{z} \boldsymbol{\Lambda}_{x}^{-1} \widetilde{\mathbf{A}}_{x 1}^{\dagger}, \\
& \mathbf{Q}_{2}=\left(\mathbf{G}_{3} \mathbf{P}_{m}\right)\left(\mathbf{G}_{2} \mathbf{P}_{m}\right)^{\dagger}=\widetilde{\mathbf{A}}_{z 1} \boldsymbol{\Phi}^{*}(\alpha) \boldsymbol{\Lambda}_{z} \boldsymbol{\Lambda}_{x}^{-1} \widetilde{\mathbf{A}}_{x 1}^{\dagger}, \\
& \mathbf{Q}_{3}=\left(\mathbf{G}_{4} \mathbf{P}_{m}\right)\left(\mathbf{G}_{1} \mathbf{P}_{m}\right)^{\dagger}=\widetilde{\mathbf{A}}_{z 1} \boldsymbol{\Phi}(\beta) \boldsymbol{\Lambda}_{z} \boldsymbol{\Lambda}_{x}^{-1} \widetilde{\mathbf{A}}_{x 1}^{\dagger}, \\
& \mathbf{Q}_{4}=\left(\mathbf{G}_{4} \mathbf{P}_{m}\right)\left(\mathbf{G}_{2} \mathbf{P}_{m}\right)^{\dagger}=\widetilde{\mathbf{A}}_{z 1} \boldsymbol{\Phi}^{*}(\alpha) \boldsymbol{\Phi}(\beta) \boldsymbol{\Lambda}_{z} \boldsymbol{\Lambda}_{x}^{-1} \widetilde{\mathbf{A}}_{x 1}^{\dagger},
\end{aligned}
$$

where $\mathbf{G}_{1}=\left[\mathbf{I}_{M-2 p}, \mathbf{0}_{(M-2 p) \times(M-2 p+2)}\right], \mathbf{G}_{2}=\left[\mathbf{0}_{(M-2 p) \times 1}\right.$, $\left.\mathbf{I}_{M-2 p}, \mathbf{0}_{(M-2 p) \times(M-2 p+1)}\right], \mathbf{G}_{3}=\left[\mathbf{0}_{(M-2 p) \times(M-2 p+1)}, \mathbf{I}_{M-2 p}\right.$, $\left.\mathbf{0}_{(M-2 p) \times 1}\right]$, and $\mathbf{G}_{4}=\left[\mathbf{0}_{(M-2 p) \times(M-2 p+2)}, \mathbf{I}_{M-2 p}\right] . \boldsymbol{\Phi}(\alpha)=$ $\operatorname{diag}\left\{e^{j \alpha_{1}}, \ldots, e^{j \alpha_{K}}\right\}, \boldsymbol{\Phi}(\beta)=\operatorname{diag}\left\{e^{j \beta_{1}}, \ldots, e^{j \beta_{K}}\right\}, \widetilde{\mathbf{A}}_{x 1} \boldsymbol{\Lambda}_{x}=$ $\mathbf{G}_{1} \widetilde{\mathbf{A}}$, and $\widetilde{\mathbf{A}}_{z 1} \boldsymbol{\Lambda}_{z}=\mathbf{G}_{3} \widetilde{\mathbf{A}}$. From (8), it is noticed that they can always hold if $\mathbf{P}_{m}$ exists (i.e., the source signals are noncoherent), while (14)-(17) in [13] cannot hold for highly correlated source signals, because $\mathbf{R}_{s s}$ cannot be approximated to a diagonal matrix for the signal correlation factor $\rho>0.3$. That is to say, (8) decreases the correlation effect.

Next, to take full advantage of the information provided by (8), construct the following augmented matrix:

$$
\begin{aligned}
& \mathbf{R}_{\mathrm{aug}}=\left[\begin{array}{c}
\mathbf{Q}_{1}, \mathbf{J}_{M-2 p} \mathbf{Q}_{4}^{*} \\
\mathbf{Q}_{2}, \mathbf{J}_{M-2 p} \mathbf{Q}_{3}^{*} \\
\mathbf{Q}_{3}, \mathbf{J}_{M-2 p} \mathbf{Q}_{2}^{*} \\
\mathbf{Q}_{4}, \mathbf{J}_{M-2 p} \mathbf{Q}_{1}^{*}
\end{array}\right] \\
&= {\left[\begin{array}{c}
\widetilde{\mathbf{A}}_{z 1} \\
\widetilde{\mathbf{A}}_{z 1} \boldsymbol{\Phi}^{*}(\alpha) \\
\widetilde{\mathbf{A}}_{z 1} \boldsymbol{\Phi}(\beta) \\
\widetilde{\mathbf{A}}_{z 1} \boldsymbol{\Phi}^{*}(\alpha) \boldsymbol{\Phi}(\beta)
\end{array}\right]\left[\widetilde{\mathbf{A}}_{x 1}^{\dagger}, \boldsymbol{\Phi}(\alpha) \boldsymbol{\Phi}^{M-2 p-1}(\beta)\right.} \\
&\left.\cdot\left(\boldsymbol{\Lambda}_{z} \Lambda_{x}^{-1} \widetilde{\mathbf{A}}_{x 1}^{\dagger}\right)^{*}\right]=\mathbf{A}_{\mathrm{aug}} \mathbf{S}_{\mathrm{aug}} .
\end{aligned}
$$

It is noticed that the construction of $\mathbf{R}_{\text {new }}$ is different from the authors' earlier work [19], where [19] just increases the effective array aperture, while the approach utilized here increases the effective array aperture and virtual snapshots simultaneously.

Similar to (6), calculate the propagator matrix $\mathbf{P}_{\text {aug }}$ corresponding to $\mathbf{R}_{\text {aug }}$ as follows:

$$
\mathbf{P}_{\text {aug }}=\left(\mathbf{R}_{1}^{\text {aug }}\left(\mathbf{R}_{1}^{\text {aug }}\right)^{H}\right)^{-1} \mathbf{R}_{1}^{\text {aug }}\left(\mathbf{R}_{2}^{\text {aug }}\right)^{H},
$$


where $\mathbf{R}_{1}^{\text {aug }}$ and $\mathbf{R}_{2}^{\text {aug }}$ are the first $K$ rows and the last ( $4 M-$ $8 p-K)$ rows of $\mathbf{R}_{\text {aug }}$. Define the modified propagator matrix $\mathbf{P}_{m}^{\text {aug }}=\left[\mathbf{I}_{K}, \mathbf{P}_{\text {aug }}\right]^{H}$ and do the calculations as follows:

$$
\begin{aligned}
& \Psi(\alpha)=\left(\mathbf{H}_{1} \mathbf{P}_{m}^{\text {aug }}\right)^{\dagger} \mathbf{H}_{2} \mathbf{P}_{m}^{\text {aug }}=\mathbf{A}_{1}^{\text {aug }} \boldsymbol{\Phi}(\alpha)\left(\mathbf{A}_{1}^{\text {aug }}\right)^{-1} \\
& \Psi(\beta)=\left(\mathbf{H}_{3} \mathbf{P}_{m}^{\text {aug }}\right)^{\dagger} \mathbf{H}_{4} \mathbf{P}_{m}^{\text {aug }}=\mathbf{A}_{1}^{\text {aug }} \boldsymbol{\Phi}(\beta)\left(\mathbf{A}_{1}^{\text {aug }}\right)^{-1}
\end{aligned}
$$

where $\mathbf{H}_{1}=\mathbf{I}_{2} \otimes\left[\mathbf{0}_{(M-2 p) \times(M-2 p)}, \mathbf{I}_{M-2 p}\right], \mathbf{H}_{2}=\mathbf{I}_{2} \otimes$ $\left[\mathbf{I}_{M-2 p}, \mathbf{0}_{(M-2 p) \times(M-2 p)}\right], \mathbf{H}_{3}=\left[\mathbf{I}_{2 M-4 p}, \mathbf{0}_{(2 M-4 p) \times(2 M-4 p)}\right]$, and $\mathbf{H}_{4}=\left[\mathbf{0}_{(2 M-4 p) \times(2 M-4 p)}, \mathbf{I}_{2 M-4 p}\right] . \mathbf{A}_{1}^{\text {aug }}$ is the first $K$ rows of $\mathbf{A}_{\text {aug }}$. It is noticed that $\Psi(\alpha)$ and $\Psi(\beta)$ have the same eigenvectors and the different eigenvalues, which is a general problem in multidimensional spectral and array signal processing [17].

To estimate azimuth and elevation simultaneously, construct $\widetilde{\boldsymbol{\Psi}}$ using the complex eigenvalue technique [17],

$$
\begin{aligned}
\widetilde{\boldsymbol{\Psi}}= & \left(\boldsymbol{\Psi}(\alpha)+\mathbf{I}_{K}\right)^{-1}\left(\boldsymbol{\Psi}(\alpha)-\mathbf{I}_{K}\right) \\
& -j\left(\boldsymbol{\Psi}(\beta)+\mathbf{I}_{K}\right)^{-1}\left(\boldsymbol{\Psi}(\beta)-\mathbf{I}_{K}\right) \\
= & \mathbf{A}_{1}^{\mathrm{aug}} \widetilde{\boldsymbol{\Phi}}\left(\mathbf{A}_{1}^{\mathrm{aug}}\right)^{-1},
\end{aligned}
$$

where $\widetilde{\boldsymbol{\Phi}}=\operatorname{diag}\left\{\tan \left(\beta_{1} / 2\right)+j \tan \left(\alpha_{1} / 2\right), \ldots, \tan \left(\beta_{K} / 2\right)+\right.$ $\left.j \tan \left(\alpha_{K} / 2\right)\right\}$. Then applying eigenvalue decomposition to $\widetilde{\boldsymbol{\Psi}}$, we can obtain $\widetilde{\Phi}$. Therefore, the azimuth and elevation angles can be estimated,

$$
\begin{aligned}
& \widehat{\phi}_{k}=\arccos \left(-\frac{\widehat{\beta}_{k} \lambda}{(2 \pi d)}\right), \\
& \widehat{\theta}_{k}=\arccos \left(-\frac{\widehat{\alpha}_{k} \lambda}{\left(2 \pi d \sin \widehat{\phi}_{k}\right)}\right),
\end{aligned}
$$

where $\widehat{\alpha}_{k}=2 \arctan [\operatorname{Im}(\widetilde{\boldsymbol{\Phi}}(k, k))]$, and $\widehat{\beta}_{K}=2 \arctan [\operatorname{Re}(\widetilde{\boldsymbol{\Phi}}(k$, $k))] . \widetilde{\Phi}(k, k)$ denotes the $k$ th row and $k$ th column element of $\widetilde{\Phi}$.

From (11) to (14), we know that the proposed method is able to estimate the angles simultaneously, which avoids the error accumulation effect that occurs in [13].

\section{Algorithm Analysis}

4.1. Limiting Performance Analysis. As shown in Section 3.1, we compensate the mutual coupling effect blindly at the cost of a few auxiliary sensors. In detail, we choose the sensors with coordinates $(m d, 0, l d)$ (where $(l, m) \in\{(1,0), \ldots,(p-$ $1,0),(M-p+1,0), \ldots,(M, 0),(0,0),(0,1), \ldots,(0, p-$ $1),(0, M-p+1), \ldots,(0, M)\})$ as the auxiliary sensors and the others as the new received array data. That is to say, the received data of $2 M-4 p+2$ sensors is used to estimate the 2D DOAs. What is more, in Section 3.2, to make (8) and (11) hold, the maximum number of sources $K_{\max } \leq M-2 p$ and the source signal correlation factor $\rho<1$ : that is, any two source signals cannot be coherent.

Besides, the choice of $p$ affects the angle estimation performance greatly. Since the smaller $p$ is, the larger array aperture and degrees of freedom can be utilized for $2 \mathrm{D}$ DOA estimation, which results in higher accuracy in angle estimation. However, $p$ originates from the approximated model of the real antenna arrays with mutual coupling [5]. The larger $p$, the smaller error for the approximation of model in (1) to real model. Hence, for real antenna arrays, there exists an optimum $p$ for angle estimation. However, the choice of optimum $p$ is a difficult task and requires complicated electromagnetic simulations and real experiments, which is beyond the scope of this paper and needs further research. In particular, according to [20-22], the real mutual coupling can be reduced via various techniques and designs. Therefore, for simplicity, we will focus on the array with fixed $p$ for the simulations in Section 5.

4.2. Computational Complexity Analysis. Before the algorithm computational complexity analysis, let us make some appointments as follows:

(i) As it is known that the complexity of complex multiplication is larger than that of complex add, we only calculate the complexity of the complex multiplication for analysis.

(ii) Since the left-multiplications of the selection matrices are just written to make the proposed method expressed well for paper writing and can be easily implemented without any multiplication operations, the computational complexity calculation does not include them, for instance, (4)-(5) and (9).

According to Section 3.2, the computational complexity of the proposed method includes construction of the propagator matrix $\mathbf{P}$ in (6): $O\left\{(2 M-4 p+2)^{2} N+2(2 M-4 p+\right.$ $\left.2) K^{2}+K^{3}+(2 M-4 p+2)(2 M-4 p+2-K) K\right\}$; construction of $\mathbf{Q}_{1}, \mathbf{Q}_{2}, \mathbf{Q}_{3}$, and $\mathbf{Q}_{4}$ in (8): $O\left\{8(M-2 p)^{2} K\right\}$; calculation of the propagator matrix $\mathbf{P}_{\text {aug }}$ in (10): $O\left\{2(2 M-4 p) K^{2}+K^{3}+\right.$ $(4 M-8 p-K)(2 M-4 p) K\} ;$ calculation of $\Psi(\alpha), \Psi(\beta)$, and $\widetilde{\Psi}$ in (11) and (12): $O\left\{2(2 M-4 p)^{2} K+2(2 M-4 p) K^{2}+4 K^{3}\right\}$; and calculation of $\widetilde{\boldsymbol{\Phi}}$ via applying eigenvalue decomposition to $\widetilde{\Psi}: O\left\{K^{3}\right\}$.

For conventional 2D DOA estimation, as $M \gg K$ and $N \gg K$, the total computational complexity of the proposed method is approximated as $O\left\{4(M-2 p+1)^{2} N+28(M-\right.$ $\left.2 p)^{2} K\right\}$. While the total computational complexity of Wu et al.'s method [12] is about $O\left\{(2 M+1)^{2} N+2 N_{\theta} N_{\phi}(p+\xi+\right.$ 1) $\left.(2 M+1)^{2}\right\}$ (where $N_{\theta}$ and $N_{\phi}$ denote the total search times within the search range of azimuth and elevation), that of Liang et al.s method [13] is about $O\left\{4(M-4)^{2} N+8(M-\right.$ $\left.4)^{2} K+(M-4-K)(M-4)^{\eta}\right\}$ (where $\eta \gg 2$ is a relatively large integer for known polynomial rooting algorithms [23]). Since $N_{\theta}, N_{\phi} \gg M$ and $\eta \gg 2$, apparently, for $p=2$, the computational complexity of the proposed method is far less than that of the two methods.

4.3. Theoretical Performance Analysis. The theoretical analysis of the proposed method is based on the matrix perturbation 
theory $[24,25]$ and the perturbed data model is given as follows:

$$
\mathbf{y}(n)=\mathbf{y}_{0}(n)+\Delta \mathbf{y}(n)=\mathbf{C A s}(n)+\mathbf{w}(n)
$$

Let $\widetilde{\mathbf{y}}(n)=\widetilde{\mathbf{y}}_{0}(n)+\Delta \widetilde{\mathbf{y}}(n)$, where $\widetilde{\mathbf{y}}_{0}(n)=\widetilde{\mathbf{A}} \mathbf{s}(n)$ and $\Delta \widetilde{\mathbf{y}}(n)=\left[\mathbf{w}_{x}^{T}(n), \mathbf{w}_{z}^{T}(n)\right]^{T}$. Define $\widetilde{\mathbf{R}}=\widetilde{\mathbf{R}}_{0}+\Delta \widetilde{\mathbf{R}}_{0}$; then the perturbation of the covariance matrix can be expressed as

$$
\begin{aligned}
\Delta \widetilde{\mathbf{R}}_{0}= & E\left\{\Delta \widetilde{\mathbf{y}}(n) \widetilde{\mathbf{y}}_{0}^{H}(n)\right\}+E\left\{\widetilde{\mathbf{y}}_{0}(n) \Delta \widetilde{\mathbf{y}}^{H}(n)\right\} \\
& +E\left\{\Delta \widetilde{\mathbf{y}}(n) \Delta \widetilde{\mathbf{y}}^{H}(n)\right\} .
\end{aligned}
$$

From (6), $\mathbf{P}=\mathbf{P}_{0}+\Delta \mathbf{P}, \widetilde{\mathbf{R}}_{1}=\widetilde{\mathbf{R}}_{1,0}+\Delta \widetilde{\mathbf{R}}_{1}$, and $\widetilde{\mathbf{R}}_{2}=\widetilde{\mathbf{R}}_{2,0}+$ $\Delta \widetilde{\mathbf{R}}_{2}$; we have $\widetilde{\mathbf{R}}_{2,0}+\Delta \widetilde{\mathbf{R}}_{2}=\left(\widetilde{\mathbf{R}}_{1,0}+\Delta \widetilde{\mathbf{R}}_{1}\right)\left(\mathbf{P}_{0}+\Delta \mathbf{P}\right)$. Neglect the second-order term $\Delta \widetilde{\mathbf{R}}_{1} \Delta \mathbf{P}$ to obtain

$$
\Delta \mathbf{P}=\widetilde{\mathbf{R}}_{1,0}^{\dagger}\left(\Delta \widetilde{\mathbf{R}}_{2}-\Delta \widetilde{\mathbf{R}}_{1} \mathbf{P}_{0}\right) .
$$

Hence, $\mathbf{P}_{m}=\mathbf{P}_{m, 0}+\Delta \mathbf{P}_{m}=\mathbf{P}_{m, 0}+\left[\mathbf{0}_{K \times K}, \Delta \mathbf{P}\right]^{H}$.

For $\mathbf{Q}_{1}, \mathbf{Q}_{2}, \mathbf{Q}_{3}$, and $\mathbf{Q}_{4}$ in (8), and similar to (17), we have

$$
\begin{aligned}
& \Delta \mathbf{Q}_{1}=\left(\mathbf{G}_{3}-\mathbf{Q}_{1,0} \mathbf{G}_{1}\right) \Delta \mathbf{P}_{m}\left(\mathbf{G}_{1} \mathbf{P}_{m, 0}\right)^{\dagger}, \\
& \Delta \mathbf{Q}_{2}=\left(\mathbf{G}_{3}-\mathbf{Q}_{2,0} \mathbf{G}_{2}\right) \Delta \mathbf{P}_{m}\left(\mathbf{G}_{2} \mathbf{P}_{m, 0}\right)^{\dagger}, \\
& \Delta \mathbf{Q}_{3}=\left(\mathbf{G}_{4}-\mathbf{Q}_{1,0} \mathbf{G}_{1}\right) \Delta \mathbf{P}_{m}\left(\mathbf{G}_{1} \mathbf{P}_{m, 0}\right)^{\dagger}, \\
& \Delta \mathbf{Q}_{4}=\left(\mathbf{G}_{4}-\mathbf{Q}_{2,0} \mathbf{G}_{2}\right) \Delta \mathbf{P}_{m}\left(\mathbf{G}_{2} \mathbf{P}_{m, 0}\right)^{\dagger} .
\end{aligned}
$$

Let $\mathbf{R}_{\mathrm{aug}}=\mathbf{A}_{\mathrm{aug}} \mathbf{S}_{\mathrm{aug}}+\Delta \mathbf{R}_{\mathrm{aug}}$; then

$$
\Delta \mathbf{R}_{\text {aug }}=\left[\begin{array}{c}
\Delta \mathbf{Q}_{1}, \mathbf{J}_{M-2 p}\left(\Delta \mathbf{Q}_{4}\right)^{*} \\
\Delta \mathbf{Q}_{2}, \mathbf{J}_{M-2 p}\left(\Delta \mathbf{Q}_{3}\right)^{*} \\
\Delta \mathbf{Q}_{3}, \mathbf{J}_{M-2 p}\left(\Delta \mathbf{Q}_{2}\right)^{*} \\
\Delta \mathbf{Q}_{4}, \mathbf{J}_{M-2 p}\left(\Delta \mathbf{Q}_{1}\right)^{*}
\end{array}\right] .
$$

Using the similar method in (17) to make the perturbation analysis of $\mathbf{P}_{\text {aug }}$ in (10), we have

$$
\Delta \mathbf{P}_{\text {aug }}=\left(\left(\mathbf{R}_{1,0}^{\text {aug }}\right)^{H}\right)^{\dagger}\left[\left(\Delta \mathbf{R}_{2}^{\text {aug }}\right)^{H}-\left(\Delta \mathbf{R}_{1}^{\text {aug }}\right)^{H} \mathbf{P}_{\text {aug }, 0}\right],
$$

where $\mathbf{P}_{\text {aug }}=\mathbf{P}_{\text {aug }, 0}+\Delta \mathbf{P}_{\text {aug }}, \mathbf{R}_{1}^{\text {aug }}=\mathbf{R}_{1,0}^{\text {aug }}+\Delta \mathbf{R}_{1}^{\text {aug }}$, and $\mathbf{R}_{2}^{\text {aug }}=$ $\mathbf{R}_{2,0}^{\text {aug }}+\Delta \mathbf{R}_{2}^{\text {aug }}$.

Hence, $\mathbf{P}_{m}^{\text {aug }}=\mathbf{P}_{m, 0}^{\text {aug }}+\Delta \mathbf{P}_{m}^{\text {aug }}=\mathbf{P}_{m, 0}^{\text {aug }}+\left[\mathbf{0}_{K \times K}, \Delta \mathbf{P}_{\text {aug }}\right]^{H}$. Further, let $\boldsymbol{\Psi}(\alpha)=\Psi_{0}(\alpha)+\Delta \Psi(\alpha), \boldsymbol{\Psi}(\beta)=\Psi_{0}(\beta)+\Delta \Psi(\beta)$; we can obtain

$$
\begin{aligned}
& \Delta \boldsymbol{\Psi}(\alpha)=\left(\mathbf{H}_{1} \mathbf{P}_{m, 0}^{\text {aug }}\right)^{\dagger}\left(\mathbf{H}_{2} \Delta \mathbf{P}_{m}^{\text {aug }}-\mathbf{H}_{1} \Delta \mathbf{P}_{m}^{\text {aug }} \boldsymbol{\Psi}_{0}(\alpha)\right), \\
& \Delta \boldsymbol{\Psi}(\beta)=\left(\mathbf{H}_{3} \mathbf{P}_{m, 0}^{\text {aug }}\right)^{\dagger}\left(\mathbf{H}_{4} \Delta \mathbf{P}_{m}^{\text {aug }}-\mathbf{H}_{3} \Delta \mathbf{P}_{m}^{\text {aug }} \boldsymbol{\Psi}_{0}(\beta)\right) .
\end{aligned}
$$

Substituting $\Delta \Psi(\alpha)$ and $\Delta \Psi(\beta)$ into (12), we use the Sherman-Morrison-Woodbury Formula [26] to obtain

$$
\begin{aligned}
\Delta \widetilde{\boldsymbol{\Psi}}= & \boldsymbol{\Psi}_{0}^{ \pm}(\alpha) \Delta \boldsymbol{\Psi}(\alpha)\left[\mathbf{I}_{K}-\boldsymbol{\Psi}_{0}^{ \pm}(\alpha) \boldsymbol{\Psi}_{0}^{-}(\alpha)\right] \\
& -j \boldsymbol{\Psi}_{0}^{ \pm}(\beta) \Delta \boldsymbol{\Psi}(\beta)\left[\mathbf{I}_{K}-\boldsymbol{\Psi}_{0}^{ \pm}(\beta) \boldsymbol{\Psi}_{0}^{-}(\beta)\right],
\end{aligned}
$$

where $\widetilde{\boldsymbol{\Psi}}=\widetilde{\boldsymbol{\Psi}}_{0}+\Delta \widetilde{\boldsymbol{\Psi}}, \boldsymbol{\Psi}_{0}^{ \pm}(\alpha)=\left(\boldsymbol{\Psi}_{0}(\alpha)+\mathbf{I}_{K}\right)^{-1}, \boldsymbol{\Psi}_{0}^{-}(\alpha)=$ $\left(\boldsymbol{\Psi}_{0}(\alpha)-\mathbf{I}_{K}\right), \boldsymbol{\Psi}_{0}^{ \pm}(\beta)=\left(\boldsymbol{\Psi}_{0}(\beta)+\mathbf{I}_{K}\right)^{-1}$, and $\boldsymbol{\Psi}_{0}^{-}(\beta)=\left(\boldsymbol{\Psi}_{0}(\beta)-\right.$ $\left.\mathbf{I}_{K}\right)$.

Calculate the first-order perturbation of the eigenvalues of $\widetilde{\boldsymbol{\Psi}}_{0}$ due to $\Delta \widetilde{\boldsymbol{\Psi}}$,

$$
\Delta \tilde{\lambda}_{k}=\mathbf{v}_{k} \Delta \widetilde{\boldsymbol{\Psi}} \mathbf{u}_{k}
$$

where $\mathbf{v}_{k}$ and $\mathbf{u}_{k}$ are the left and right orthonormal eigenvectors of $\widetilde{\Psi}_{0}$ associated with $\widetilde{\lambda}_{k}$; that is, $\widetilde{\Psi}_{0}=\left[\mathbf{u}_{1}, \ldots, \mathbf{u}_{K}\right]$. $\operatorname{diag}\left\{\tilde{\lambda}_{1}, \ldots, \widetilde{\lambda}_{K}\right\} \cdot\left[\mathbf{v}_{1}^{T}, \ldots, \mathbf{v}_{K}^{T}\right]^{T}$.

Further, using the relationship that $\alpha_{k}=$ $2 \arctan \left(\operatorname{Im}\left(\widetilde{\lambda}_{k}\right)\right), \beta_{k}=2 \arctan \left(\operatorname{Re}\left(\widetilde{\lambda}_{k}\right)\right)$, we can obtain

$$
\begin{aligned}
& \Delta \alpha_{k}=2 \cos ^{2} \frac{\alpha_{k}}{2} \operatorname{Im}\left\{\Delta \tilde{\lambda}_{k}\right\}, \\
& \Delta \beta_{k}=2 \cos ^{2} \frac{\beta_{k}}{2} \operatorname{Re}\left\{\Delta \tilde{\lambda}_{k}\right\} .
\end{aligned}
$$

Given $\phi_{k}=\arccos \left(-\beta_{k} \lambda /(2 \pi d)\right), \theta_{k}=\arccos \left(-\alpha_{k} \lambda /\right.$ $\left.\left(2 \pi d \sin \phi_{k}\right)\right)$, the perturbations of azimuth and elevation angles are

$$
\begin{aligned}
\Delta \phi_{k} & =\frac{\partial \phi_{k}}{\partial \beta_{k}} \Delta \beta_{k}=C_{\phi \beta} \Delta \beta_{k} \\
\Delta \theta_{k} & =\left[\frac{\partial \theta_{k}}{\partial \alpha_{k}}, \frac{\partial \theta_{k}}{\partial \phi_{k}}\right]\left[\begin{array}{l}
\Delta \alpha_{k} \\
\Delta \phi_{k}
\end{array}\right]=C_{\theta \alpha} \Delta \alpha_{k}+C_{\theta \phi} \Delta \phi_{k},
\end{aligned}
$$

where $C_{\phi \beta}=\lambda /(2 \pi d) / \sqrt{1-\left(\beta_{k} \lambda /(2 \pi d)\right)^{2}}, C_{\theta \alpha}=\lambda /$ $\left(2 \pi d \sin \phi_{k}\right) / \sqrt{1-\left(\alpha_{k} \lambda /\left(2 \pi d \sin \phi_{k}\right)\right)^{2}}, C_{\theta \phi}=-\alpha_{k} \lambda \cos \phi_{k} /$ $\left(2 \pi d \sin ^{2} \phi_{k}\right) / \sqrt{1-\left(\alpha_{k} \lambda /\left(2 \pi d \sin ^{2} \phi_{k}\right)\right)^{2}}$.

Therefore, the mean-squared error of estimated DOAs is

$$
\begin{aligned}
\operatorname{var}\left(\Delta \phi_{k}\right)= & C_{\phi \beta}^{2} \operatorname{var}\left(\Delta \beta_{k}\right) \\
= & 2 \cos ^{2} \frac{\beta_{k}}{2} C_{\phi \beta}^{2} \operatorname{var}\left(\operatorname{Re}\left\{\Delta \tilde{\lambda}_{k}\right\}\right) \\
\operatorname{var}\left(\Delta \theta_{k}\right)= & C_{\theta \alpha}^{2} \operatorname{var}\left(\Delta \alpha_{k}\right)+C_{\theta \phi}^{2} \operatorname{var}\left(\Delta \phi_{k}\right) \\
= & 2 \cos ^{2} \frac{\alpha_{k}}{2} C_{\theta \alpha}^{2} \operatorname{var}\left(\operatorname{Im}\left\{\Delta \tilde{\lambda}_{k}\right\}\right) \\
& +2 \cos ^{2} \frac{\beta_{k}}{2} C_{\phi \beta}^{2} C_{\theta \phi}^{2} \operatorname{var}\left(\operatorname{Re}\left\{\Delta \tilde{\lambda}_{k}\right\}\right) .
\end{aligned}
$$

\section{Simulation Results}

In this section, the $2 \mathrm{D}$ DOA estimation performance of the proposed method is verified through simulation experiments in comparison with its theoretical analysis, Wu et al's method [12], Liang et al.'s method [13], and the Cramer-Rao bound (CRB) [27]. Without loss of generality, we assume that $d=$ $\lambda / 2$, all source signals have the same power $\sigma_{s}^{2}$, and the signal to noise ratio (SNR) is defined as $10 \log _{10}\left(\sigma_{s}^{2} / \sigma_{w}^{2}\right)$. The search ranges of azimuth and elevation angles for Wu et al.s method are $\left[0^{\circ}, 180^{\circ}\right]$ with interval $0.01^{\circ}$. In order to measure the 
overall performance of estimating the azimuth and elevation angles, define the root-mean-square-error (RMSE) of the 2D DOA estimations from $V$ Monte Carlo trials as

$$
\mathrm{RMSE}=\sqrt{\frac{1}{K V} \sum_{v=1}^{V} \sum_{k=1}^{K}\left(\left(\widehat{\theta}_{k}^{(v)}-\theta_{k}\right)^{2}+\left(\widehat{\phi}_{k}^{(v)}-\phi_{k}\right)^{2}\right)}
$$

In addition, to evaluate the performance of the proposed method under different correlation factor $\rho$ between the incident signals, we generate two source signals $\mathbf{s}_{1}(n)$ and $\mathbf{s}_{2}(n)$ with determined $\rho$ as follows:

$$
\mathbf{s}_{2}(n)=\mathbf{s}_{1}(n)+\sqrt{1-|\rho|^{2}} \mathbf{s}_{3}(n)
$$

where $\mathbf{s}_{1}(n)$ is uncorrelated with $\mathbf{s}_{3}(n)$. The magnitude of $\rho$ varies from 0 to 1 . Specially, for $\rho=0$, we usually call two source signals uncorrelated or independent. While for $|\rho|=$ 1 , two source signals are called coherent.

Since Wu et al.s method and Liang et al.s method can also be used for $p \geq 2$ with a few modifications, for simplicity, we choose $p=2$ for the following simulation examples. The mutual coupling coefficients are $c_{1}=0.4528-0.3764 j, c_{2}=$ $0.1085+0.0987 j$, and $c_{3}=0.1828-0.3076 j$ associated with distances $d, 2 d$, and $\sqrt{2} d$.

Example 1 (estimation performance versus SNR). In the first example, the performance of the proposed method with respect to the SNR under different source signal correlations is investigated. Two sources are placed at $\left(115^{\circ}, 65^{\circ}\right)$ and $\left(95^{\circ}, 80^{\circ}\right)$. The number of subarray elements and the number of snapshots are fixed at $M=8$ and $N=500$. SNR varies from $-10 \mathrm{~dB}$ to $30 \mathrm{~dB}$ with interval $-2.5 \mathrm{~dB}$. 500 Monte Carlo trials have been conducted for every fixed SNR. Figures 2 and 3 show the RMSEs of 2D DOA estimations for $\rho=0$ and $\rho=0.6$, that is, the uncorrelated and high correlated cases.

As we can see, the proposed method yields better 2D DOA estimation performance than Liang et al's method for $\mathrm{SNR} \geq-5 \mathrm{~dB}$ and its experimental performance coincides with the theoretical analysis well for $\mathrm{SNR} \geq 0 \mathrm{~dB}$, which verifies the correctness of the proposed method and its theoretical analysis. Specially, for $\rho=0.6$, the increase of angle estimation accuracy with the proposed method to Liang et al.'s method is greater than that of $\rho=0$, which proves that the proposed method can decrease the correlation between highly correlated source signals. Besides, from Figure 2, since the proposed method estimates angles simultaneously, it has better angle estimation performance than Liang et al's method which divides the angle estimations into two sequential steps and results in the error accumulation effect.

However, to estimate 2D DOAs with less time, the proposed method utilizes some array elements as auxiliary sensors and some of the array aperture is lost; hence, the angle estimation performance of proposed method is worse than that of Wu et al.'s method for both uncorrelated and correlated cases.

Example 2 (estimation performance versus the number of snapshots). In this example, we concentrate on the 2D DOA

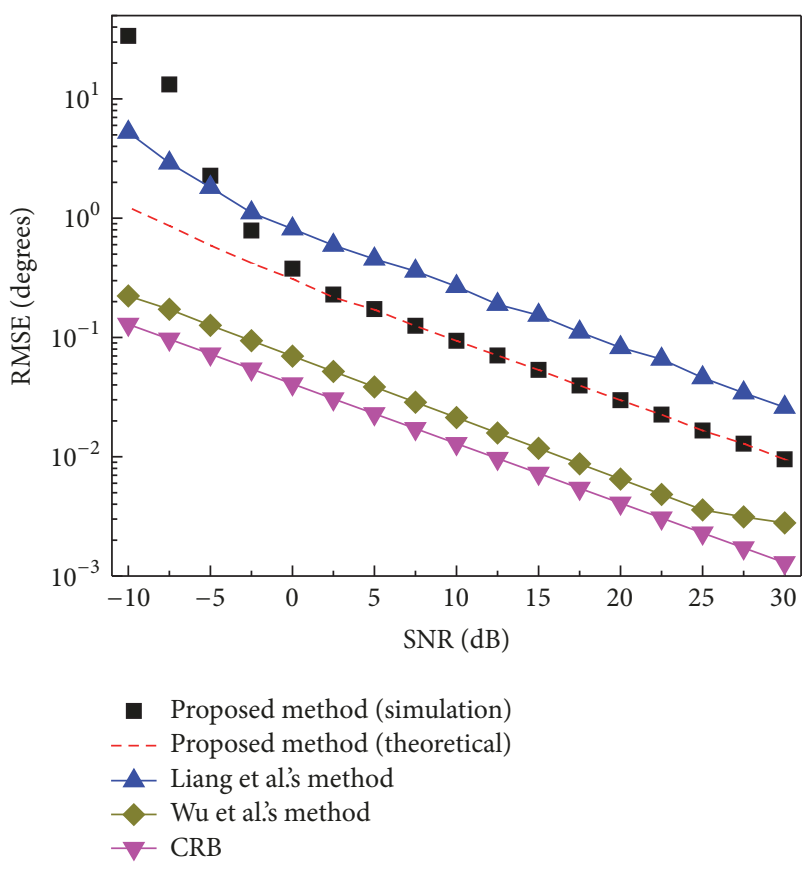

FIGURE 2: RMSE versus SNR, $M=8, N=500, \rho=0$.

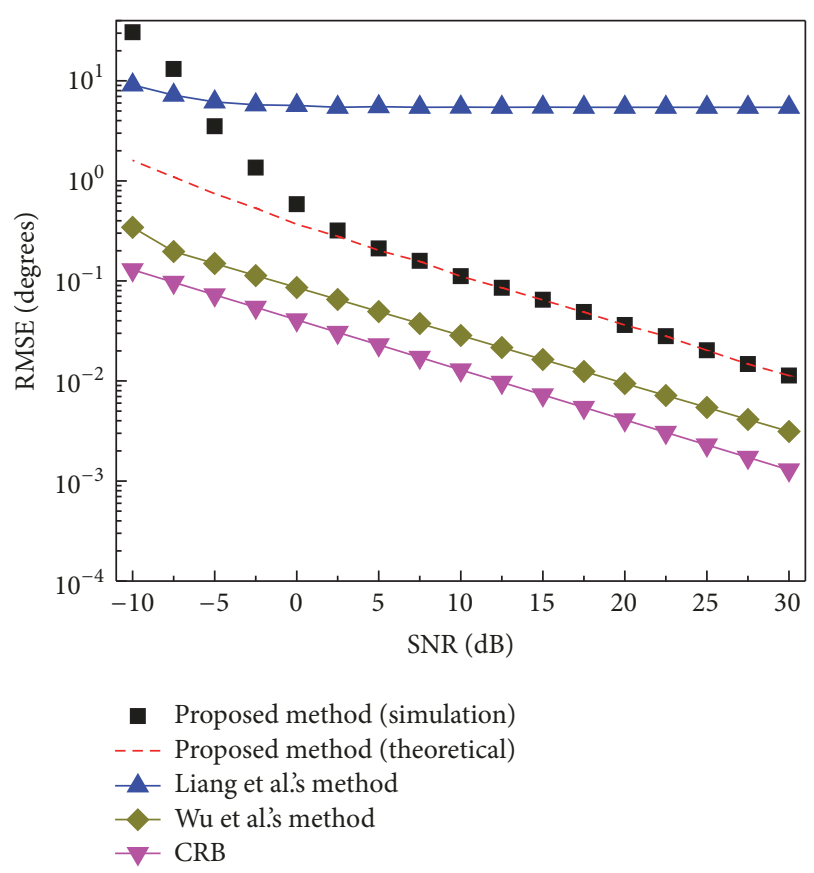

Figure 3: RMSE versus SNR, $M=8, N=500, \rho=0.6$.

estimation performance of the proposed method versus the number of snapshots with different $\rho$ 's. All simulation conditions are the same as Example 1 except that SNR is fixed at $10 \mathrm{~dB}$ and $N$ ranges from 100 to 10000 . The angle estimation results are shown in Figures 4 and 5.

We can know that the angle estimation performance of the proposed method outperforms that of Liang et al's method for different N's under SNR $=10 \mathrm{~dB}$ case. What is 


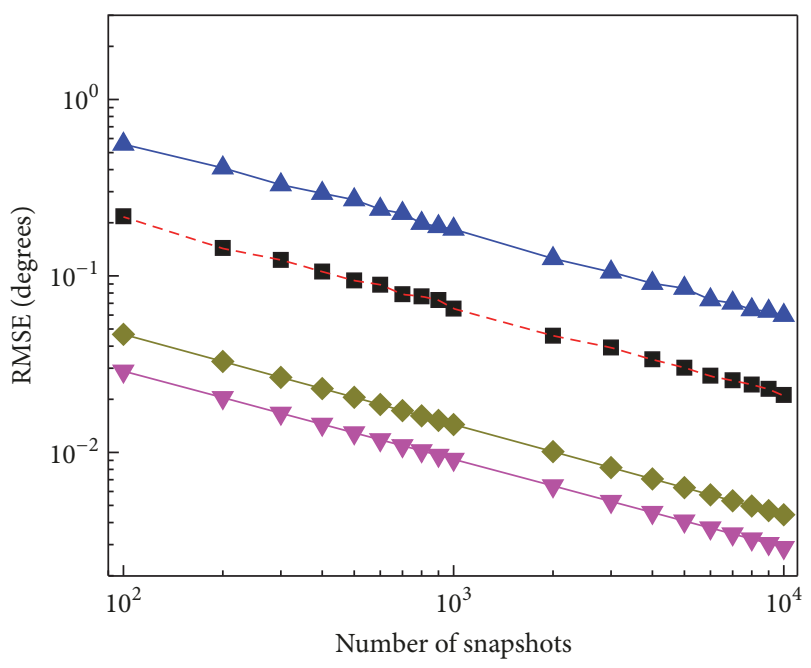

- Proposed method (simulation)

- - Proposed method (theoretical)

— Liang et al.'s method

- Wu et al.s method

$\rightarrow \mathrm{CRB}$

Figure 4: RMSE versus $N, M=8, \mathrm{SNR}=10 \mathrm{~dB}, \rho=0$.

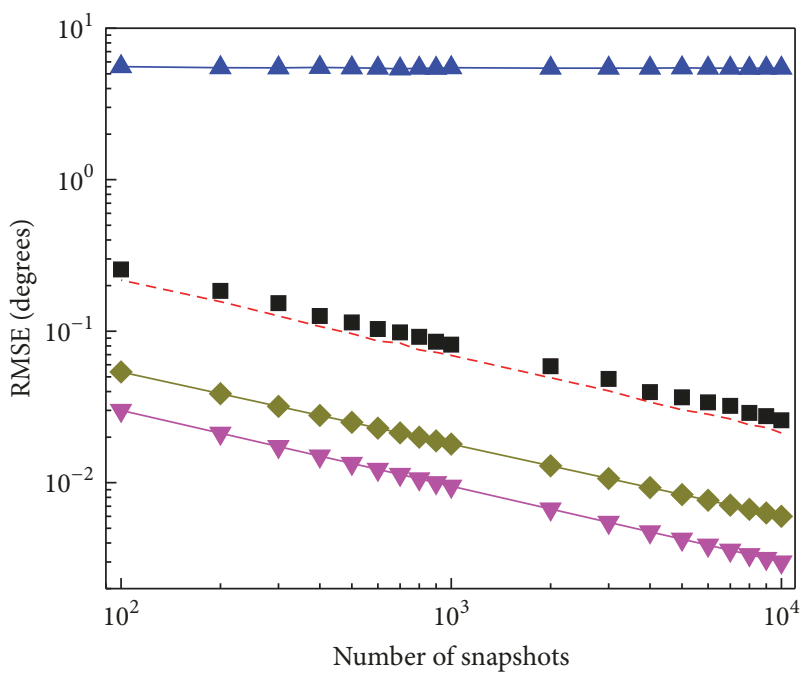

- Proposed method (simulation)

- - - Proposed method (theoretical)

- Liang et al's method

$\checkmark$ Wu et al's method

$\rightarrow \mathrm{CRB}$

FIGURE 5: RMSE versus $N, M=8, \mathrm{SNR}=10 \mathrm{~dB}, \rho=0.6$.

more, for $\rho=0.6$, the proposed method works well, while Liang et al.'s method does not, which verifies the decorrelation performance of the proposed method. The theoretical performance is very close to the experimental results under two different $\rho$ 's. For $\rho=0.6$, the experimental results are always slightly higher than the theoretical results because the proposed method can only decrease the correlation effect not eliminate it.

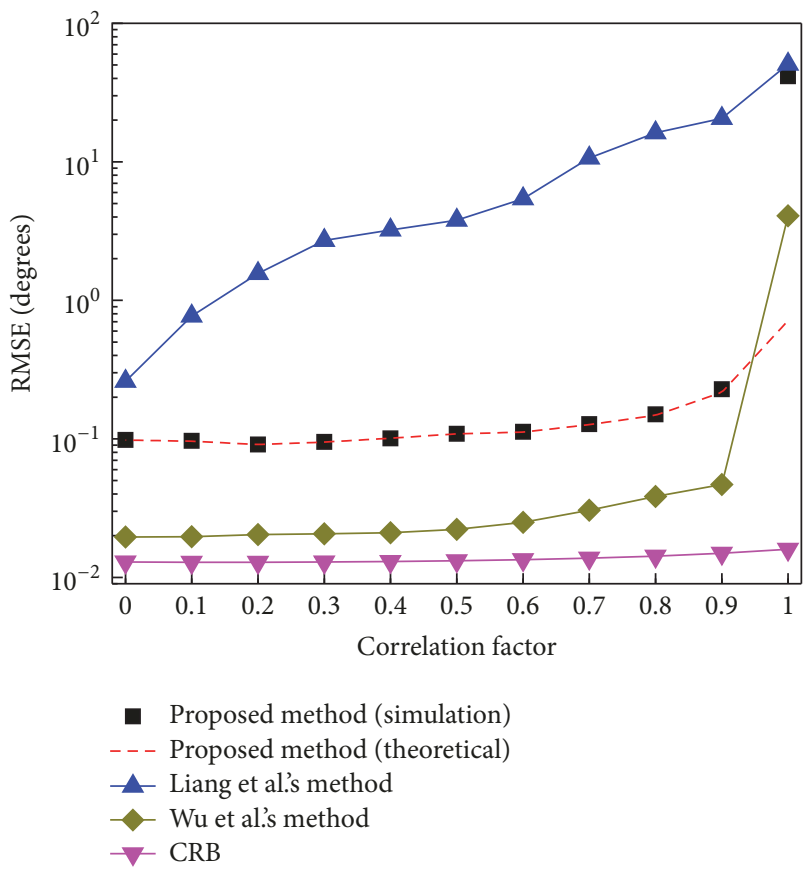

FIGURE 6: RMSE versus correlation factor, $M=8$, SNR $=10 \mathrm{~dB}, N=$ 500.

Similar to Example 1, from Figures 4 and 5, we know that the 2D DOA estimation performance of the proposed method is still worse than that of Wu et al's method owing to the loss in array aperture.

Example 3 (estimation performance versus the correlation factor). In this example, we consider the angle estimation performance against the correlation factor $\rho$ and the simulation settings are similar to that of Example 1 except that SNR is equal to $10 \mathrm{~dB}$, and the correlation factor $\rho$ varies from 0 (uncorrelated) to 1 (coherent). The results are shown in Figure 6.

It is noticed that the $2 \mathrm{D} \mathrm{DOA}$ estimation performance of the proposed method and $\mathrm{Wu}$ et al's method are both more robust to the source signal correlation than that of Liang et al.s method for $0 \leq \rho \leq 0.9$. Importantly, similar to Wu et al.'s method, the proposed method can work well with extremely high correlation factor: that is, $\rho=0.9$. In addition, both of the three methods cannot work at coherent case: that is, $\rho=1$, which is an open problem for research in the future.

Example 4 (runtime versus the number of subarray elements). In this example, we investigate the runtime of the proposed method with respect to $M$. All simulation conditions are the same as Example 1 except that $\mathrm{SNR}=10 \mathrm{~dB}$ and $M$ ranges from 8 to 96 with interval 8 . The implementation of Liang et al's method utilizes the Symbolic Toolbox, sym2poly, and roots function in MATLAB. The measurements of cpu runtime for these three methods are shown in Figure 7, and Figure 8 gives the ratios of the runtime of the proposed method to those of the other two methods. 


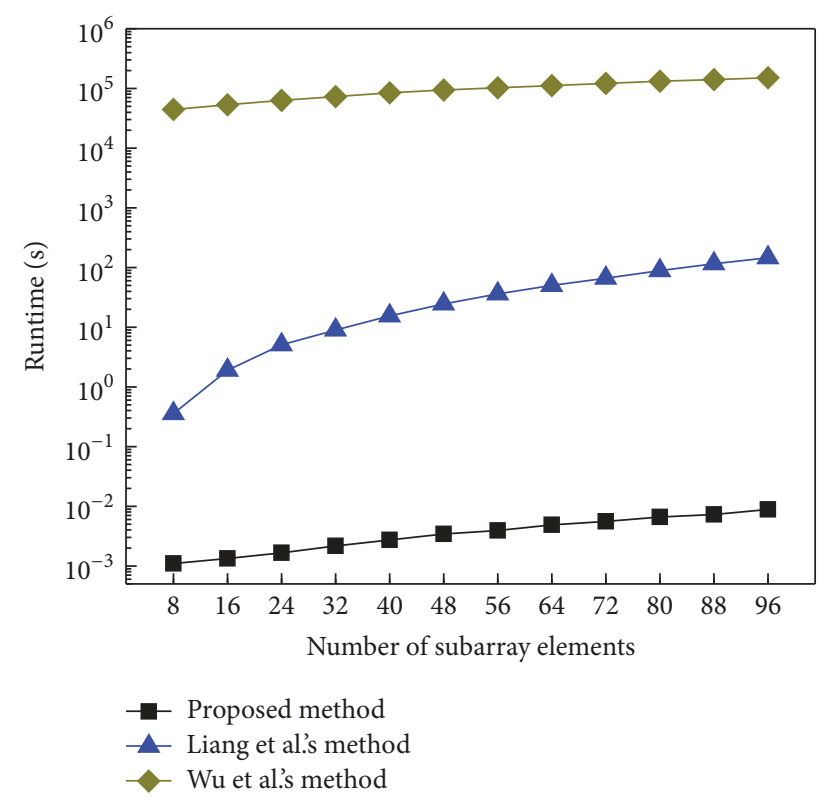

FIGURE 7: Runtime versus the number of subarray elements, SNR = $10 \mathrm{~dB}, N=500, \rho=0.6$.

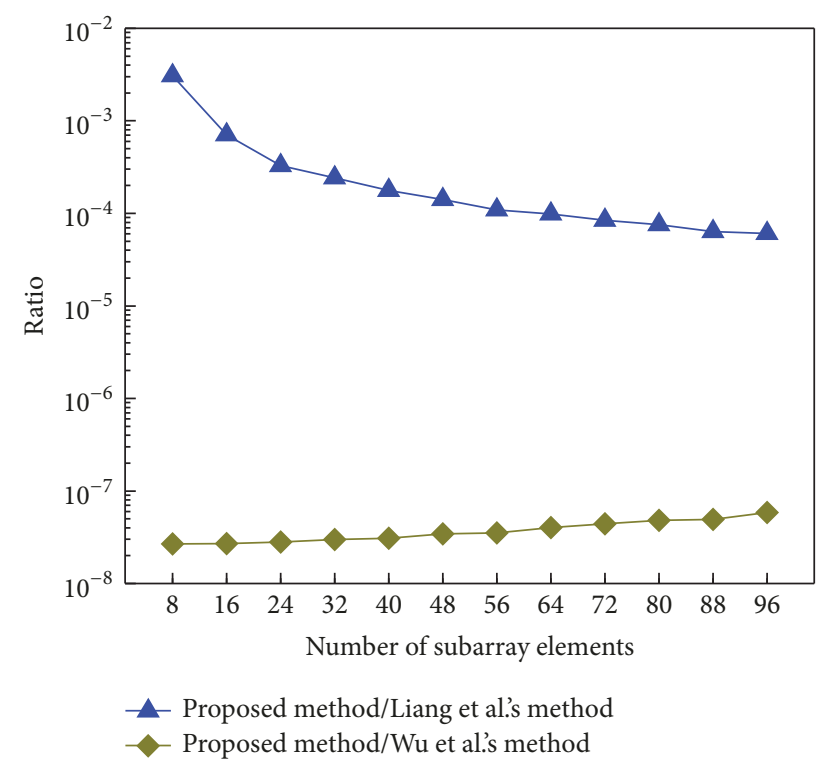

FIGURE 8: Ratio versus the number of subarray elements, SNR = $10 \mathrm{~dB}, N=500, \rho=0.6$.

Apparently, the proposed method is much more computationally efficient than Liang et al's method and Wu et al's method. What is more, the runtime of the proposed method is less than $10^{-6}$ of Wu et al.'s and $10^{-2}$ of Liang et al.s, which indicates that the proposed method is a significant improvement of Wu et al's method and Liang et al's method in computation time. Besides, as shown in Figure 8, with the increase in the number of subarray elements, the ratio of the cpu runtime of the proposed method to that of Liang et al's method decreases greatly. The reason is that their computational complexity expressions, neglecting the lower order terms and the constant coefficients including $N$, can be expressed approximately as $O\left\{M^{2}\right\}$ and $O\left\{M^{\eta+1}\right\}(\eta \gg 2)$. Therefore, when $M$ increases, the ratio decreases greatly as shown in Figure 8.

Overall, from Figures $2-8$, the proposed method can estimate 2D DOAs against mutual coupling under highly correlated cases, which is similar to the ability of Wu et al's method. Importantly, the proposed method has the lowest computational complexity among the three methods. Besides, the angle estimation performance of the proposed method is outperformed by $\mathrm{Wu}$ et al.s method in all cases, which results from the utilization of RARE technique guaranteeing less loss in array aperture and degrees of freedom than the auxiliary sensor method used by the proposed method.

\section{Conclusions}

In this paper, we have developed a computationally efficient 2D DOA estimation method for L-shaped array with unknown mutual coupling and its explicit expressions of theoretical performance for angle estimations have also been derived. Simulation results and theoretical analysis have verified the effectiveness of the proposed method and shown that the proposed method can handle highly correlated source signals with lower computational complexity and also has fine 2D DOA estimation performance against coupling effect. However, the proposed method has assumed that some elements are auxiliaries which is not consistent with the cases in real scenarios and results in larger estimation errors for deployment cases. Hence, further research on real application test is needed.

\section{Conflicts of Interest}

The authors declare that there are no conflicts of interest regarding the publication of this paper.

\section{Acknowledgments}

This work was supported in part by the National HighTech R\&D Program of China (863 Program) under Grant 2015AA80XXX86H, in part by the Fundamental Research Funds for the Central Universities of China under Grant JB140203, and in part by the China Postdoctoral Science Foundation under Grant 2017M623123.

\section{References}

[1] H. L. Van Trees, Detection, Estimation, and Modulation Theory, Optimum Array Processing, John Wiley \& Sons, 2004.

[2] G.-Q. Zhao, Principle of Radar Countermeasure, Xidian University Press, 2nd edition, 2012.

[3] I. J. Gupta and A. A. Ksienski, "Effect of mutual coupling on the performance of adaptive arrays," IEEE Transactions on Antennas and Propagation, vol. 31, no. 5, pp. 785-791, 1983.

[4] H. T. Hui, "A new definition of mutual impedance for application in dipole receiving antenna arrays," IEEE Antennas and Wireless Propagation Letters, vol. 3, no. 1, pp. 364-367, 2004. 
[5] B. Friedlander and A. J. Weiss, "Direction finding in the presence of mutual coupling," IEEE Transactions on Antennas and Propagation, vol. 39, no. 3, pp. 273-284, 1991.

[6] K. R. Dandekar, H. Ling, and G. Xu, "Effect of mutual coupling on direction finding in smart antenna applications," Electronics Letters, vol. 36, no. 22, pp. 1889-1891, 2000.

[7] C. M. S. See, "Sensor array calibration in the presence of mutual coupling and unknown sensor gains and phases," IEEE Electronics Letters, vol. 30, no. 5, pp. 373-374, 1994.

[8] W. Chen, J. P. Lie, B. P. Ng, T. Wang, and M. H. Er, "Joint gain/phase and mutual coupling array calibration technique with single calibrating source," International Journal of Antennas and Propagation, vol. 2012, Article ID 625165, 8 pages, 2012.

[9] M. Lin and L. Yang, "Blind calibration and DOA estimation with uniform circular arrays in the presence of mutual coupling," IEEE Antennas and Wireless Propagation Letters, vol. 5, no. 1, pp. 315-318, 2006.

[10] Z. Ye and C. Liu, "2-D DOA estimation in the presence of mutual coupling," IEEE Transactions on Antennas and Propagation, vol. 56, no. 10, pp. 3150-3158, 2008.

[11] C. Liu, Z. Ye, and Y. Zhang, "Autocalibration algorithm for mutual coupling of planar array," Signal Processing, vol. 90, no. 3, pp. 784-794, 2010.

[12] B. Wu, H. Chen, and C. H. Yang, "Study of DOA estimation and self-calibration algorithm for L-shaped array in the presence of mutual coupling," Acta Electron. Sinica, vol. 38, no. 6, pp. 13161322, 2010.

[13] J. Liang, X. Zeng, W. Wang, and H. Chen, "L-shaped arraybased elevation and azimuth direction finding in the presence of mutual coupling," Signal Processing, vol. 91, no. 5, pp. 1319-1328, 2011.

[14] H. Wu, C. Hou, H. Chen, W. Liu, and Q. Wang, "Direction finding and mutual coupling estimation for uniform rectangular arrays," Signal Processing, vol. 117, pp. 61-68, 2015.

[15] M. Rübsamen and A. B. Gershman, "Sparse array design for azimuthal direction-of-arrival estimation," IEEE Transactions on Signal Processing, vol. 59, no. 12, pp. 5957-5969, 2011.

[16] Y. Hua, T. K. Sarkar, and D. D. Weiner, "An L-shaped array for estimating 2-D directions of wave arrival," IEEE Transactions on Antennas and Propagation, vol. 39, no. 2, pp. 143-146, 1991.

[17] Y. Hua and K. Abed-Meraim, "Techniques of Eigenvalues Estimation and Association," Digital Signal Processing, vol. 7, no. 4, pp. 253-259, 1997.

[18] S. Marcos, A. Marsal, and M. Benidir, "The propagator method for source bearing estimation," Signal Processing, vol. 42, no. 2, pp. 121-138, 1995.

[19] Y.-Y. Dong, C.-X. Dong, J. Xu, and G.-Q. Zhao, "Computationally efficient 2-D DOA estimation for 1-shaped array with automatic pairing," IEEE Antennas and Wireless Propagation Letters, vol. 15, pp. 1669-1672, 2016.

[20] C.-Y. Chiu, C.-H. Cheng, R. D. Murch, and C. R. Rowell, "Reduction of mutual coupling between closely-packed antenna elements," IEEE Transactions on Antennas and Propagation, vol. 55, no. 6, pp. 1732-1738, 2007.

[21] Y. Aslan and A. Yarovoy, "Reduction of mutual coupling between closely spaced patch antennas using dielectric stratification technique," in Proceedings of the 2017 47th European Microwave Conference (EuMC), pp. 248-251, Nuremberg, Germany, October 2017.

[22] S. Gupta, Z. Briqech, A. R. Sebak, and T. Ahmed Denidni, "Mutual-Coupling Reduction Using Metasurface Corrugations for $28 \mathrm{GHz}$ MIMO Applications," IEEE Antennas and Wireless Propagation Letters, vol. 16, pp. 2763-2766, 2017.

[23] K. V. Rangarao and S. Venkatanarasimhan, "Gold-MUSIC: A variation on MUSIC to accurately determine peaks of the spectrum," IEEE Transactions on Antennas and Propagation, vol. 61, no. 4, pp. 2263-2268, 2013.

[24] F. Li, H. Liu, and R. J. Vaccaro, "Performance analysis for DOA estimation algorithms: unification, simplification, and observations," IEEE Transactions on Aerospace and Electronic Systems, vol. 29, no. 4, pp. 1170-1184, 1993.

[25] H. Chen, C. Hou, W.-P. Zhu et al., "ESPRIT-like twodimensional direction finding for mixed circular and strictly noncircular sources based on joint diagonalization," Signal Processing, vol. 141, pp. 48-56, 2017.

[26] H. V. Henderson and S. R. Searle, "On deriving the inverse of a sum of matrices," SIAM Review. A Publication of the Society for Industrial and Applied Mathematics, vol. 23, no. 1, pp. 53-60, 1981.

[27] S. M. Kay, Fundamentals of Statistical Signal Processing: Estimation Theory, Prentice-Hall, 1993. 


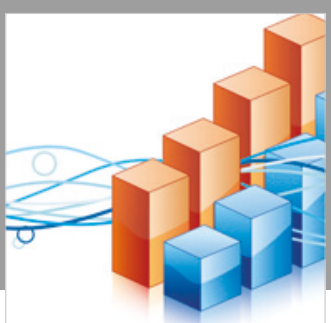

Advances in

Operations Research

\section{-n-m}
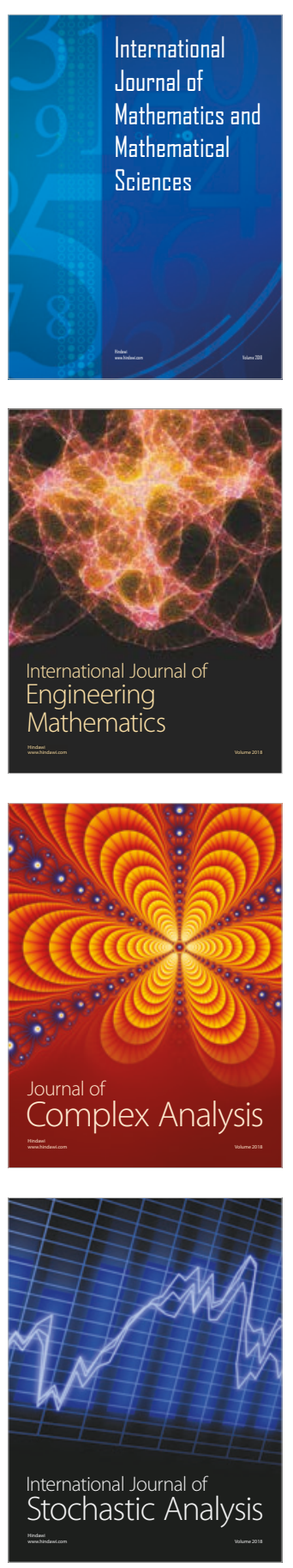
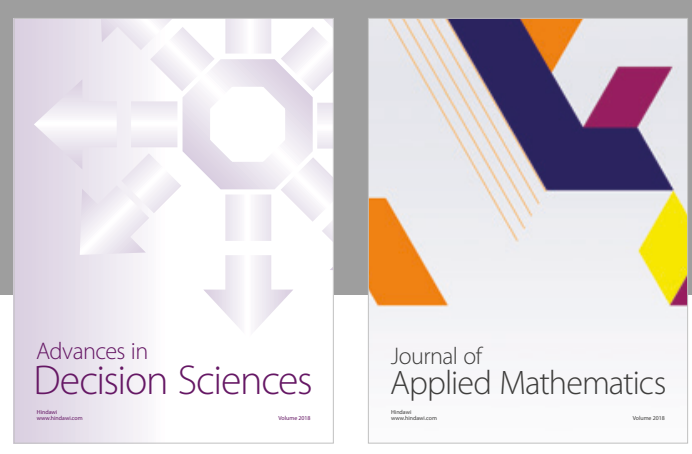

Journal of

Applied Mathematics
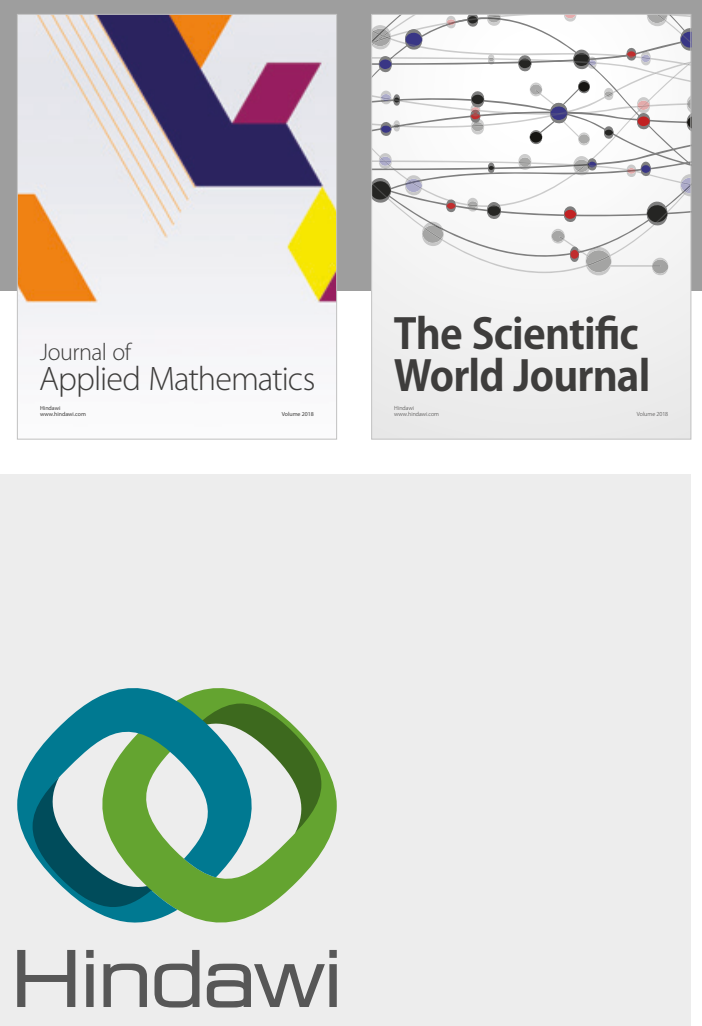

Submit your manuscripts at

www.hindawi.com

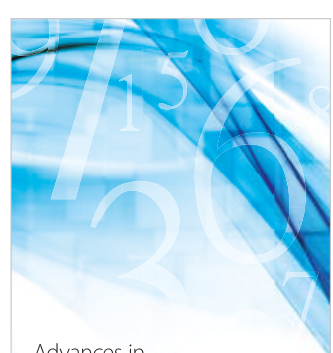

Advances in
Numerical Analysis
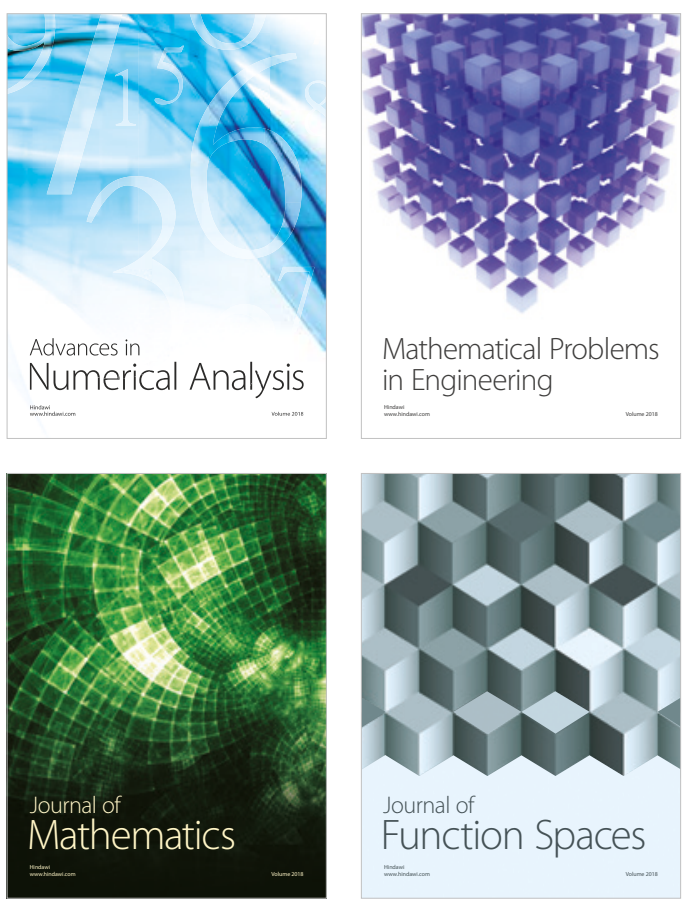

Mathematical Problems in Engineering

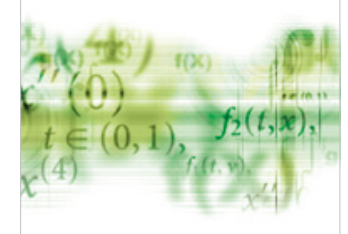

International Journal of

Differential Equations

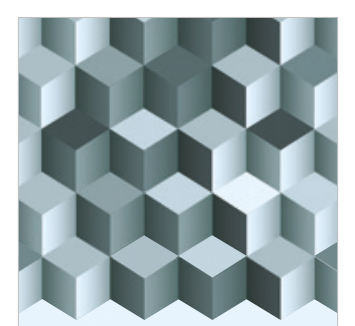

Journal of

Function Spaces

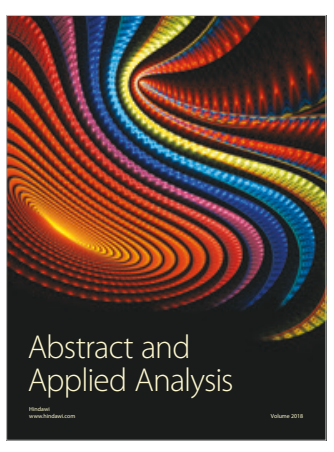

The Scientific

World Journal

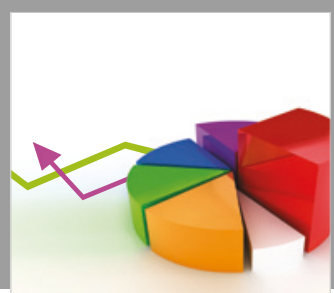

Journal of

Probability and Statistics
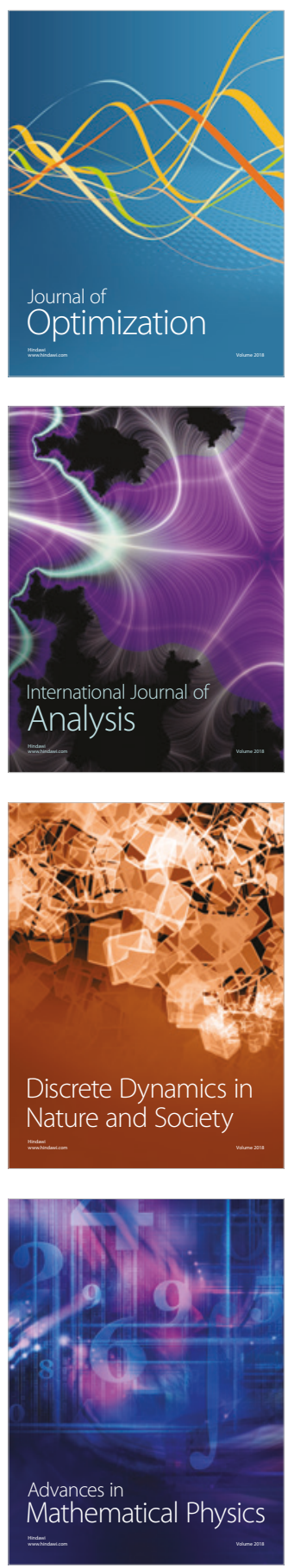